\title{
Prevalência da apneia obstrutiva do sono em trabalhadores de turno: uma revisão sistemática
}

\author{
Prevalence of obstructive sleep apnea in shift workers: \\ a systematic review
}

Yuri Saho Sakamoto ${ }^{1}$ Fernanda Porto-Sousa ${ }^{1}$ Cristina Salles ${ }^{1}$
${ }^{1}$ Escola Bahiana de Medicina e Saúde Pública. Av. Dom João VI 274, Brotas. 40285-001 Salvador BA Brasil.

yurisaho@yahoo.com

\begin{abstract}
The obstructive sleep apnea (OSA) is one of the most common sleep disorders and is associated with increased risk for obesity, hypertension and cardiovascular event. The present study aimed to investigate the prevalence of OSA in shift workers. The systematic literature review was performed using the descriptors "sleep apnea" and "shift work", in the databases PubMed, PubMed Central, Biblioteca Virtual em Saúde, Web of Science and Scopus, including studies that presented the frequency of OSA in shift workers; published between 2004 and 2014; in English, Portuguese or Spanish; only with human beings, older than 18 years old; using the polysomnography. Review articles or those including participants with previous comorbidities (except overweight/obesity), treated for sleep disorders or pregnant women were excluded. From 1,428 studies identified, four were included in the analysis, resulting in 819 participants, with a predominance of men. The prevalence of OSA in shift workers varied from $14.3 \%$ to $38.1 \%$, higher than that estimated for the general population, suggesting an important association with shift work and the need for prevention, diagnostic and intervention on the possible negative impacts of working range on the health of shift workers.
\end{abstract}

Key words Sleep apnea, Obstructive, Shift work, Prevalence, Workers
Resumo A apneia obstrutiva do sono (AOS) é um dos distúrbios do sono mais frequentes, associada ao risco aumentado para obesidade, hipertensão e evento cardiovascular. O presente estudo buscou investigar a prevalência da AOS em trabalhadores de turno. Foi realizada a revisão sistemática da literatura, utilizando-se os descritores "sleep apnea" AND "shift work", nas bases de dados do PubMed Central, Biblioteca Virtual em Saúde, Web of Science e Scopus, incluindo-se artigos que apresentaram a frequência da AOS em trabalhadores de turno, publicados entre 2004 e 2014, em inglês, português ou espanhol, apenas com seres humanos, maiores de 18 anos, utilizando polissonografia. Artigos de revisão ou com participantes com comorbidades prévias (exceto sobrepeso/obesidade), tratados para doença do sono ou gestantes foram excluídos. Dos 1.428 artigos identificados, quatro foram incluídos para análise, totalizando 819 participantes, com predomínio do sexo masculino. A prevalência da AOS em trabalhadores de turno variou de $14,3 \%$ a $38,1 \%$, superior à estimada para a população geral, sugerindo associação importante com o trabalho de turno e a necessidade de prevenção, diagnóstico e intervenção sobre possivivis impactos negativos da escala de trabalho na saúde desses trabalhadores.

Palavras-chave Apneia do Sono tipo Obstrutiva, Trabalho em Turnos, Prevalência, Trabalhadores 


\section{Introdução}

Observa-se a frequência crescente do trabalho de turno na sociedade atual ${ }^{1}$. Nos Estados Unidos, aproximadamente 15 milhões de americanos trabalham em regime de turnos ou escalas irregulares $^{2}$. No Brasil, estima-se que $10 \%$ ou mais dos trabalhadores atuam à noite ou em turnos ${ }^{1}$.

Distúrbios do sono apresentam alta probabilidade de associação com o trabalho de turno ${ }^{3}$ e a apneia obstrutiva do sono (AOS) é um dos distúrbios respiratórios do sono mais comuns ${ }^{4}$, merecendo especial atenção no manejo da saúde desses trabalhadores, pois pode atuar como fator de risco para hipertensão arterial sistêmica ${ }^{5} \mathrm{e}$ contribuir para eventos cardiovasculares ${ }^{4,6}$ e síndrome metabólica ${ }^{5}$.

Prejuízos pessoais, sociais e econômicos decorrentes de distúrbios do sono em trabalhadores de turno são elevados, considerando-se os impactos na saúde física e psíquica do trabalhador e sua família, redução na produtividade, acidentes de trabalho e custos com segurança e saúde pública $^{3,7}$. Tendo em vista que dados de prevalência da AOS podem fundamentar iniciativas de prevenção baseadas nos riscos para a doença, ações de diagnóstico precoce e tratamento adequado, o presente trabalho teve como objetivo investigar a prevalência da apneia obstrutiva do sono em trabalhadores de turno.

\section{Métodos}

Foi realizada a revisão sistemática de literatura, com pesquisa nas bases de dados eletrônicas PubMed, PubMed Central, Biblioteca Virtual em Saúde, Web of Science e Scopus. Como descritores, utilizou-se os termos do Medical Subject Headings (MeSH) e Descritores em Ciências da Saúde (DeCS) correspondentes a "sleep apnea" AND "shift work", obtendo-se o detalhamento de busca: ("sleep apnoea" [All Fields] OR "sleep apnea syndromes"[MeSH Terms] OR ("sleep"[All Fields] AND "apnea"[All Fields] AND "syndromes"[All Fields]) OR "sleep apnea syndromes"[All Fields] OR ("sleep" [All Fields] AND "apnea”[All Fields]) OR "sleep apnea”[All Fields]) AND (shift[All Fields] AND ("work"[MeSH Terms] OR “work”[All Fields])).

Adicionalmente, realizou-se a busca manual nas referências dos artigos selecionados.

Foram incluídos estudos que abordaram a frequência da AOS em trabalhadores de turno; publicados a partir de 01/01/2004 e disponibilizados nas bases de dados até 18/12/2014, visando contemplar os trabalhos mais atualizados, abrangendo o período de 10 anos; nos idiomas inglês, português ou espanhol; cujos participantes apresentavam idade igual ou superior a 18 anos; realizados somente com seres humanos; utilizando a polissonografia como método diagnóstico da AOS. Foram excluídos artigos de revisão; cujos participantes apresentavam comorbidades prévias (exceto sobrepeso e obesidade), em tratamento para distúrbios do sono ou gestantes.

Os trabalhos foram identificados por dois autores que, de forma independente, avaliaram seus títulos e resumos, triando-os conforme os critérios de inclusão e exclusão. Os trabalhos sem resumo ou com resumo insuficiente para avaliação, não excluídos pelo título, foram também triados para leitura do texto completo. Cada autor avaliou separadamente os textos completos, para inclusão nesta revisão sistemática. A busca manual seguiu o mesmo princípio de seleção. Nos casos de divergências, um terceiro autor foi consultado.

Foram coletados os seguintes dados dos artigos selecionados: título; autor(es); ano de publicação; país de origem; revista científica de publicação; idioma; palavras-chave; objetivo(s); desenho de estudo; método; período de realização; critérios de inclusão e exclusão; tamanho da amostra; critério(s) diagnóstico(s) da AOS; idade dos participantes; sexo dos participantes; escala de trabalho; prevalência da AOS na amostra; Índice de Apneia e Hipopneia (IAH); características clínicas dos trabalhadores de turno com AOS; outros resultados da pesquisa; e conclusões.

A análise da qualidade dos artigos baseou-se no Strengthening the Reporting of Observational Studies in Epidemiology (STROBE) ${ }^{8}$. Os artigos incluídos na revisão sistemática foram analisados em consenso pelos autores, atribuindo-se uma classificação em relação a cada item do STROBE: item integralmente atendido; parcialmente atendido; ou não ficou claro o cumprimento do item. Aqueles que atenderam aos critérios de qualidade na presente revisão sistemática obtiveram pelo menos 11 itens integral ou parcialmente atendidos.

A apresentação dos resultados desta revisão sistemática seguiu as recomendações do Preferred Reporting Items for Systematic Reviews and Meta -Analyses (PRISMA).

\section{Resultados}

Do total de 1.598 trabalhos encontrados nas bases de dados eletrônicas, 296 eram duplicatas, obtendo-se 1.302 estudos. Foram excluídos 1.298 
artigos e, a partir dos quatro estudos selecionados, realizou-se a busca manual, totalizando 126 artigos, nenhum elegível para a presente revisão sistemática. Permaneceram quatro estudos que atendiam as condições para inclusão na presente revisão sistemática (Figura 1 ).
Os artigos selecionados eram observacionais, de corte transversal. As características gerais dos estudos incluídos na revisão sistemática estão resumidas na Tabela 1.

Santos et al. ${ }^{10}$ buscaram avaliar o sono diurno e noturno de motoristas de ônibus que tra-

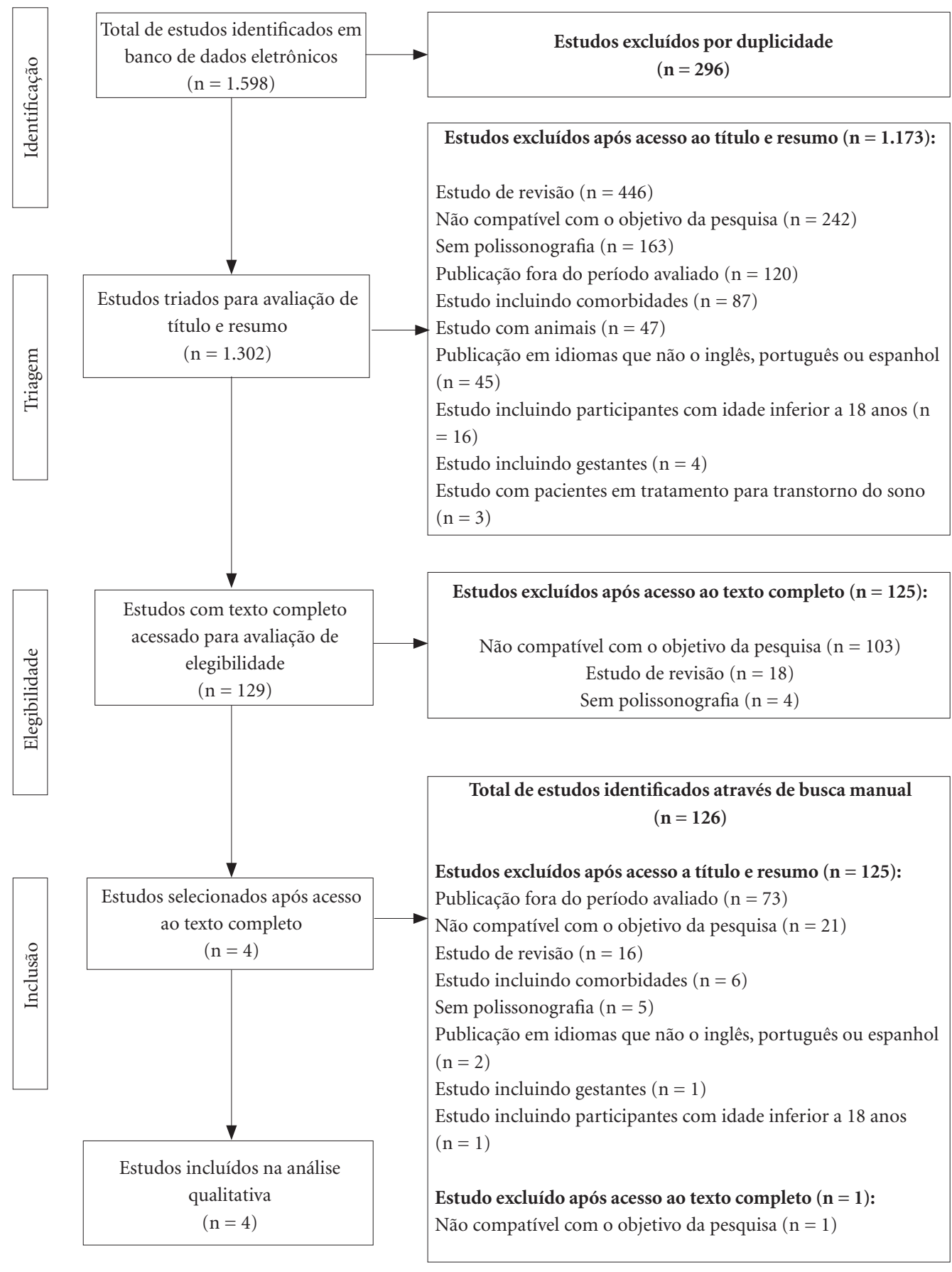

Figura 1. Fluxograma de identificação, triagem, elegibilidade e inclusão de estudos na revisão sistemática. 
Tabela 1. Características gerais dos estudos selecionados, ordenados por ano de publicação.

\begin{tabular}{|c|c|c|c|c|c|c|}
\hline Autores & País, ano & $\begin{array}{l}\text { Desenho de } \\
\text { estudo }\end{array}$ & $\mathbf{N}$ & $\begin{array}{c}\text { Sexo } \\
\text { masculino } \\
(\%)\end{array}$ & $\begin{array}{l}\text { Idade } \\
\text { média } \\
\text { (anos) }\end{array}$ & Escala de trabalho \\
\hline Santos et al..$^{10}$ & $\begin{array}{l}\text { Brasil, } \\
2004\end{array}$ & $\begin{array}{l}\text { Estudo analítico, } \\
\text { corte transversal. }\end{array}$ & 32 & 100 & $42,5 \pm 7,1$ & $\begin{array}{l}\text { Turno de } 8 \text { horas de trabalho, } \\
\text { após o mínimo de } 12 \text { horas de } \\
\text { descanso, com um ou dois dias } \\
\text { de folga, após quatro ou cinco } \\
\text { dias de trabalho. }\end{array}$ \\
\hline Klawe et al. ${ }^{11}$ & $\begin{array}{l}\text { Polônia, } \\
2005\end{array}$ & $\begin{array}{l}\text { Estudo analítico, } \\
\text { corte transversal. }\end{array}$ & 21 & NI & $47,1 \pm 3,2$ & $\begin{array}{l}\text { Escala tipo } \\
\text { fast-rotating. }\end{array}$ \\
\hline Koyama et al. ${ }^{12}$ & $\begin{array}{l}\text { Brasil, } \\
2012\end{array}$ & $\begin{array}{l}\text { Estudo analítico, } \\
\text { corte transversal. }\end{array}$ & 745 & 100 & $35,6 \pm 9,6$ & NI \\
\hline $\begin{array}{l}\text { Geiger-Brown } \\
\text { et al. }{ }^{13}\end{array}$ & $\begin{array}{l}\text { Estados } \\
\text { Unidos, } \\
2013\end{array}$ & $\begin{array}{l}\text { Estudo } \\
\text { exploratório, } \\
\text { corte transversal. }\end{array}$ & 21 & 0 & $40 \pm 13$ & $\begin{array}{l}\text { Turno de } 12 \text { horas em tempo } \\
\text { integral ( } 50 \% \text { da amostra em } \\
\text { turnos fixos noturnos, } 25 \% \\
\text { em rodízios de turno diurno e } \\
\text { noturno; e } 25 \% \text { em turnos fixos } \\
\text { diurnos). }\end{array}$ \\
\hline
\end{tabular}

balhavam em turnos, e a presença de sonolência diurna e noturna e distúrbios do sono nesses profissionais. Foram avaliados 32 motoristas que atuavam em turnos, em uma empresa brasileira de transporte interestadual, através da polissonografia. O Multiple Sleep Latency Test (MSLT) foi realizado após a polissonografia, para avaliação de sonolência excessiva. As variáveis polissonográficas foram comparadas através do teste $t$-Student, e, pelo teste de McNemar analisou-se o movimento periódico de pernas, IAH, ronco e sonolência, considerando-se $p \leq 0,05$ como estatisticamente significante ${ }^{10}$.

Klawe et al. ${ }^{11}$ buscaram identificar a presença de distúrbios respiratórios do sono em trabalhadores de turno com redução do sono diário, associado à privação cumulativa de sono. Para tanto, realizaram a polissonografia noturna em 21 policiais que atuavam em turnos, após um dia de trabalho de turno e uma noite normal de sono. Os resultados foram comparados aos do grupo controle, composto por 21 policiais com faixa etária compatível, trabalhando no mesmo ambiente, submetidos à polissonografia nas mesmas condições. Aplicou-se o teste de Wilcoxon para análise estatística, considerando-se $p<0,05$ como estatisticamente significante ${ }^{11}$.

Koyama et al. ${ }^{12}$ tiveram como objetivo avaliar a prevalência e possíveis fatores de risco para a síndrome de AOS em trabalhadores ferroviários brasileiros. Incluíram 745 trabalhadores de uma empresa brasileira de transporte ferroviário, sem critérios de exclusão. Além da coleta de dados antropométricos e gerais, os autores aplicaram o Epworth Sleepiness Scale (ESS) e o Questionário de Berlim. Todos os participantes foram submetidos à polissonografia noturna para avaliação de seu padrão de sono e diagnóstico da AOS. Pelo teste de qui-quadrado, foram analisadas as variáveis categóricas, considerando-se $\alpha<0,05$ como estatisticamente significante. Os fatores de risco para AOS foram categorizados e explorados através da curva Receiver Operating Characteristic para variáveis contínuas de idade, circunferência abdominal e tempo como trabalhador de turno. Determinou-se os odds ratios ( $95 \%$ de IC) e realizou-se a regressão logística para identificar fatores de risco independentes significantes para $\mathrm{AOS}^{12}$.

Geiger-Brown et al. ${ }^{13}$ buscaram descrever a prevalência de sintomas de distúrbios respiratórios do sono em enfermeiros, e avaliar a validade do Questionário de Berlim para triagem de apneia do sono em um grupo com privação parcial crônica de sono. O estudo incluiu 21 enfermeiras, selecionadas entre participantes de outro estudo maior ${ }^{14}$, submetidas à polissonografia noturna, cujos achados foram sintetizados, utilizando-se as médias (desvio padrão) e medianas. A performance do Questionário de Berlim foi avaliada a partir dos cálculos de prevalência, sensibilidade, especificidade, valor preditivo positivo, valor preditivo negativo e razão de verossimilhança ${ }^{13}$.

Dois estudos analisados na presente revisão sistemática foram realizados exclusivamente com homens ${ }^{10,12}$, um foi realizado somente com mulheres ${ }^{13}$ e um não especificou o sexo dos partici- 
pantes $^{11}$. Pelo menos $94,9 \%$ da amostra da presente revisão foi composta pelo sexo masculino.

A idade média dos participantes variou de $35,6 \pm 9,6$ anos $^{12}$ a $47,1 \pm 3,2$ anos $^{11}$. Apenas um estudo $^{12}$ especificou a média de idade dos trabalhadores de turno diagnosticados com AOS: $38,5 \pm 10,1$ anos. Comparando-os com o grupo de trabalhadores de turno em geral $(35,6 \pm 9,6$ anos) e com o grupo de trabalhadores de turno sem diagnóstico da AOS (34 $\pm 8,9$ anos), esses autores observaram maior risco para AOS nos trabalhadores com mais de 37 anos em sua amostra $(p<0,001)^{12}$.

Quanto à qualidade dos estudos selecionados, o estudo de Koyama et al. ${ }^{12}$ obteve melhor desempenho, cumprindo todos os itens propos- tos pelo STROBE: vinte itens cumpridos integralmente e dois itens cumpridos parcialmente (Figura 2).

Santos et al. ${ }^{10}$ destacaram que não foi possível controlar a quantidade de turnos noturnos de trabalho, antes da polissonografia diurna dos motoristas pesquisados, e o primeiro exame foi sempre realizado à noite. Desta forma, a influência da ordem dos turnos na aplicação da polissonografia sobre os resultados não foi avaliada. Adicionalmente, o ambiente artificial do laboratório pode não ter reproduzido com fidelidade o sono em ambiente familiar para os participantes ${ }^{10}$.

Klawe et al. ${ }^{11}$ também consideraram que o momento da polissonografia pode ter influenciado os resultados em seu estudo, considerando

\begin{tabular}{|c|c|c|c|c|c|}
\hline Tópico & Item & Santos et al. ${ }^{10}$ & Klawe et al. ${ }^{11}$ & Koyama et al. ${ }^{12}$ & $\begin{array}{c}\text { Geiger-Brown } \\
\text { et al. } .^{13}\end{array}$ \\
\hline Título e Resumo & 1 & 0 & 0 & 0 & 0 \\
\hline \multicolumn{6}{|l|}{ Introdução } \\
\hline Contexto/Justificativa & 2 & - & - & - & - \\
\hline Objetivos & 3 & ? & ? & 0 & - \\
\hline \multicolumn{6}{|l|}{ Métodos } \\
\hline Desenho do Estudo & 4 & 0 & 0 & - & 0 \\
\hline Contexo (setting) & 5 & - & 0 & - & 0 \\
\hline Participantes & 6 & 0 & 0 & - & 0 \\
\hline Variáveis & 7 & 0 & o & - & o \\
\hline Fontes de dados/Mensuração & 8 & $\bullet$ & 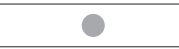 & $\bullet$ & 0 \\
\hline Viés & 9 & - & 0 & $\bullet$ & 0 \\
\hline Tamanho do Estudo & 10 & 0 & 0 & $\bullet$ & 0 \\
\hline Variáveis Quantitativas & 11 & - & $\bullet$ & - & ? \\
\hline Métodos Estatísticos & 12 & 0 & 0 & $\bullet$ & 0 \\
\hline \multicolumn{6}{|l|}{ Resultados } \\
\hline Participantes & 13 & 0 & 0 & - & 0 \\
\hline Dados descritivos & 14 & 0 & - & $\bullet$ & 0 \\
\hline Desfecho & 15 & - & $\bullet$ & $\bullet$ & 0 \\
\hline Resultados principais & 16 & 0 & 0 & $\bullet$ & 0 \\
\hline Outras Análises & 17 & - & 0 & $\bullet$ & 0 \\
\hline \multicolumn{6}{|l|}{ Discussão } \\
\hline Resultados principais & 18 & $\bullet$ & $\bullet$ & $\bullet$ & 0 \\
\hline Limitações & 19 & - & 0 & $\bullet$ & ? \\
\hline Interpretação & 20 & - & $\bullet$ & - & 0 \\
\hline Generalização & 21 & - & 0 & - & - \\
\hline \multicolumn{6}{|l|}{ Outras Informações } \\
\hline Financiamento & 22 & 0 & $\bullet$ & $\bullet$ & 0 \\
\hline
\end{tabular}

Item integralente atendido pelo artigo Item parcialmente atendido pelo artigo

O Não ficou claro o cumprimento do item pelo artigo

Figura 2. Avaliação da qualidade dos estudos selecionados, com base nos itens essenciais da iniciativa Strengthening the Reporting of Observational Studies in Epidemiology (STROBE) ${ }^{8}$. 
que, caso o exame fosse realizado na noite imediatamente posterior à noite de trabalho, poderia fornecer dados quanto ao efeito direto do trabalho noturno sobre a qualidade do sono dos participantes.

Koyama et al..$^{12}$ apresentaram como limitação o uso de questionários autoaplicáveis para obterem informações que habitualmente são omitidas pelos trabalhadores, incluindo tabagismo, etilismo e sonolência excessiva.

Geiger-Brown et al. ${ }^{13}$ apresentaram três limitações principais em seu estudo: o tamanho reduzido da amostra; o risco de viés de seleção da amostra, pois buscaram obter escores positivos e negativos do Questionário de Berlim para alcançar o poder de análise no estudo; e o possível "efeito de primeira-noite" da polissonografia. Este efeito é observado durante a primeira noite do exame polissonográfico, devido à influência de diferentes fatores sobre a qualidade do sono, tais como a falta de familiaridade com o ambiente artificial do laboratório, a ansiedade do paciente por ser observado durante o sono, e o desconforto pelo uso de eletrodos, cabos e outros equipamentos ${ }^{15}$.

Na presente revisão sistemática, as amostras de trabalhadores de turno avaliados através da polissonografia resultaram no $\mathrm{n}$ total de 819 participantes. A prevalência da AOS em trabalhadores de turno pesquisados nos estudos variou de $14,3 \%{ }^{13}$ a $38,1 \%{ }^{11}$ (Tabela 2).

As principais características clínicas e polissonográficas dos trabalhadores de turno de cada estudo selecionado são resumidas na Tabela $2 \mathrm{e}$ detalhadas a seguir.

Geiger-Brown et al. ${ }^{13}$ não apresentaram o Índice de Massa Corporal (IMC) dos participantes em sua pesquisa, porém respostas do Questionário de Berlim indicaram que $33 \%$ da sua amostra apresentava IMC $\geq 30 \mathrm{~kg} / \mathrm{m}^{2}$. O IMC médio dos demais participantes da pesquisa correspondeu à faixa de sobrepeso e obesidade (IMC $\geq 25 \mathrm{~kg} /$ $\left.\mathrm{m}^{2}\right)^{10-12}$, mas apenas o estudo de Koyama et al. ${ }^{12}$ apresentou o IMC médio específico dos trabalhadores de turno diagnosticados com AOS: 27,7 $\pm 4,4 \mathrm{~kg} / \mathrm{m}^{2}$. Este estudo indicou que $69,5 \%$ dos participantes em geral apresentavam sobrepeso ou obesidade, sendo que, entre os trabalhadores sem diagnóstico da AOS, $63,2 \%$ apresentaram IMC $\geq 25 \mathrm{~kg} / \mathrm{m}^{2}$, enquanto $77 \%$ dos trabalhadores com diagnóstico da AOS apresentavam essa condição. A diferença entre a prevalência de sobrepeso e obesidade em trabalhadores sem AOS e com AOS, identificada por Koyama et al. ${ }^{12}$, foi

Tabela 2. Características clínicas e polissonográficas da amostra estudada.

\begin{tabular}{|c|c|c|c|c|c|}
\hline \multirow[b]{2}{*}{ Referências } & \multirow{2}{*}{$\begin{array}{c}\text { Trabalhadores } \\
\text { diagnosticados } \\
\text { com AOS: } \\
\text { N (\%) }\end{array}$} & \multicolumn{2}{|r|}{ Amostra geral } & \multicolumn{2}{|c|}{ Trabalhadores com AOS } \\
\hline & & $\begin{array}{l}\text { IMC médio } \\
\left(\mathrm{Kg} / \mathrm{m}^{2}\right)\end{array}$ & $\begin{array}{l}\text { Frequência de outros } \\
\text { distúrbios do sono }\end{array}$ & $\begin{array}{c}\text { IMC } \\
\text { médio } \\
\left(\mathrm{Kg} / \mathrm{m}^{2}\right)\end{array}$ & Hábitos de vida \\
\hline Santos et al. ${ }^{10}$ & $12(37,5)$ & $26 \pm 4,9$ & $\begin{array}{l}\text { MPM: } 28 \% \text { durante o sono } \\
\text { diurno e } 18 \% \text { no sono } \\
\text { noturno. } \\
\text { Ronco: } 50 \% \text { durante o sono } \\
\text { diurno e } 35 \% \text { durante o sono } \\
\text { noturno. } \\
\text { Sonolência excessiva: } 42 \% \\
\text { durante o dia e } 38 \% \text { durante } \\
\text { a noite. }\end{array}$ & NI & NI \\
\hline Klawe et al. ${ }^{11}$ & $8(38,1)$ & $28,1 \pm 2,1$ & NI & NI & NI \\
\hline $\begin{array}{l}\text { Koyama et } \\
\text { al. }{ }^{12}\end{array}$ & $261(35)$ & $26,7 \pm 4,1$ & NI & $27,7 \pm 4,4$ & $\begin{array}{l}\text { Tempo médio de } \\
\text { trabalho de turno } \\
\text { de } 14,3 \pm 9,1 \text { anos } \\
(\mathrm{p}<0,001) ; 54,7 \% \\
\text { consumiam álcool } \\
(\mathrm{p}=0,4) ; 9,5 \% \text { eram } \\
\text { tabagistas ( } \mathrm{p}=0,24) .\end{array}$ \\
\hline $\begin{array}{l}\text { Geiger- } \\
\text { Brown et al. }{ }^{13}\end{array}$ & $3(14,3)$ & NI & $\underline{\text { MPM: }}$ 9,5\% & NI & $\mathrm{NI}$ \\
\hline
\end{tabular}


estatisticamente significante, com $p<0,001^{12}$. O IMC $\geq 25 \mathrm{~kg} / \mathrm{m}^{2}$ foi apontado por esses autores como fator associado ao risco para AOS, com odds ratio de 1,86 (1,31-2,64), pela análise univariada, e 1,56 (1,04-2,34), pela análise multivariada.

O IAH médio da amostra geral do estudo de Geiger-Brown et al. ${ }^{13}$ correspondeu a 2,3 $\pm 5,4$ eventos obstrutivos/hora de sono (ev/h), não sendo fornecido o IAH médio dos trabalhadores de turno com AOS. Nos demais estudos ${ }^{10-12}$, não foi apresentado o IAH médio dos trabalhadores de turno em geral ou daqueles diagnosticados com AOS.

Dois trabalhos incluíram a investigação de outros distúrbios relacionados ao sono entre os participantes ${ }^{10,13}$. Apenas Koyama et al. ${ }^{12}$ investigaram o tempo médio de trabalho de turno, o consumo de álcool e o tabagismo, entre trabalhadores de turno com AOS.

\section{Discussão}

$\mathrm{Na}$ presente revisão sistemática, obteve-se a amostra total de 819 trabalhadores de turno, observando-se a prevalência da AOS, diagnosticada através da polissonografia basal, variando de $14,3 \%$ a $38,1 \%$ nos estudos analisados. Este resultado é superior às estimativas dessa doença para a população adulta em geral, onde a prevalência pode variar entre $2 \%$ e $14 \%{ }^{16-18}$.

Young et al. ${ }^{16}$, partindo da polissonografia noturna em trabalhadores de 30 a 60 anos, estimaram a prevalência da AOS em 4\% para os homens e $2 \%$ para as mulheres. Kim et al. ${ }^{17}$, utilizando o critério de $\mathrm{IAH} \geq 5 \mathrm{ev} / \mathrm{h}$ associado à sonolência diurna, observaram que 4,5\% dos homens e 3,2\% das mulheres de sua amostra apresentavam a síndrome de AOS. Peppard et al. ${ }^{18}$, analisando dados de 1.520 participantes do Wisconsin Sleep Cohort Study, estimaram a prevalência da AOS em indivíduos entre 30 e 70 anos em cerca de $13 \%$ em homens e $6 \%$ em mulheres $(\mathrm{IAH} \geq 15 \mathrm{ev} / \mathrm{h})$, e $14 \%$ em homens e $5 \% \mathrm{em}$ mulheres (IAH $\geq 5 \mathrm{ev} / \mathrm{h}$, com sintomas de sonolência diurna). Comparando-se a prevalência estimada da AOS em trabalhadores de turno, nesta revisão sistemática, com aquela estimada para a população em geral, reforça-se a hipótese de que há associação significativa entre trabalhado de turno e AOS. Neste sentido, trabalhadores de turno se configuram como profissionais expostos ao risco possivelmente aumentado para AOS, doença relacionada às repercussões importantes para a saúde, produtividade e qualidade de vida.
Os critérios diagnósticos da AOS utilizados nos estudos selecionados na presente revisão sistemática divergiram entre si, apesar de adotarem a polissonografia, padrão-ouro para se determinar a presença dessa síndrome ${ }^{19}$. Em 2009, a diretriz da American Academy of Sleep Medicine (AASM) para avaliação, manejo e cuidados de longo prazo para AOS, em adultos, estabeleceu os seguintes critérios para diagnóstico da doença: $\mathrm{IAH} \geq 15 \mathrm{ev} / \mathrm{h}$, ou IAH $\geq 5 \mathrm{ev} / \mathrm{h}$ associado aos sintomas relacionados ao sono, que podem ser investigados a partir de instrumentos validados, como o $\mathrm{ESS}^{19}$. Para determinar o IAH, a diretriz estabeleceu a polissonografia como método diagnóstico objetivo de escolha ${ }^{19}$, seguindo-se as especificações estabelecidas pela AASM, que foram atualizadas em $2012^{20}$.

Dentre os artigos publicados antes de 2009, Klawe et al. ${ }^{11}$ consideraram que os trabalhadores de turno com IAH $\geq 5 \mathrm{ev} / \mathrm{h}$ apresentavam AOS. Santos et al. ${ }^{10}$ classificaram os participantes como apresentando distúrbios respiratórios do sono quando IAH $\geq 5 \mathrm{ev} / \mathrm{h}$, no sono diurno e noturno, o que observaram em $38 \%$ da amostra. Uma proporção considerável de motoristas de ônibus neste estudo foi diagnosticada com a síndrome de $\mathrm{AOS}^{10}$. Apesar de Geiger-Brown et al. ${ }^{13}$ terem realizado o estudo após 2009, consideraram o IAH $\geq$ 5 ev/h para diagnosticar AOS leve. Já Koyama et al. ${ }^{12}$ seguiram as recomendações da AASM, adotando como critérios para AOS: IAH $\geq 15 \mathrm{ev} / \mathrm{h}$; ou o IAH $\geq 5 \mathrm{ev} / \mathrm{h}$ e escore a partir de 11 pontos pelo ESS ou roncos ou relatos de apneia durante o sono; além de utilizarem o Questionário de Berlim, para avaliar risco para AOS.

Dois estudos ${ }^{10,12}$ avaliaram trabalhadores brasileiros e identificaram a AOS em 37,5\% e 35\% de suas amostras, respectivamente. Um estudo de base populacional, realizado na população geral adulta do estado de São Paulo, utilizando entrevistas presenciais e polissonografia noturna, identificou que, dentre 1.042 participantes, 341 $(32,8 \%)$ obtiveram o diagnóstico da doença, também superior ao sugerido na literatura ${ }^{21}$. A maior frequência da AOS encontrada nos trabalhadores de turno, na presente revisão sistemática, pode ter sido influenciada por similaridades entre as amostras, com consequente elevação da prevalência da doença, comparada com outras populações.

A amostra selecionada, nesta revisão sistemática, foi composta predominantemente pelo sexo masculino, o que pode ter relação com a ocupação dos trabalhadores das pesquisas. Por exemplo, quase 95\% dos profissionais pesquisados atuavam na área de transporte, atividades 
nas quais o sexo masculino tende a ser maioria ${ }^{22}$. Este fator pode ter contribuído para a maior prevalência da AOS observada na amostra, uma vez que a prevalência da doença na população em geral tende a ser maior entre os homens, na proporção de 2:1 a 2,8:1 em relação às mulheres ${ }^{16,18}$.

A idade dos participantes do presente estudo pode ter contribuído para a maior frequência da AOS, comparada à população em geral. Além disso, Koyama et al. ${ }^{12}$ identificaram que a idade média de trabalhadores ferroviários que atuavam em turnos diagnosticados com AOS era superior à observada entre aqueles que não apresentaram a AOS, e sugeriram a associação entre idade e prevalência da doença, com risco aumentado em trabalhadores mais velhos e, especialmente, com idade acima de 37 anos. Esses achados são corroborados por trabalhos que apontam a frequência crescente de doenças respiratórias do sono com a idade, presentes em mais de $50 \%$ da população acima de 65 anos $^{23}$.

Um estudo desenvolvido na Pensilvânia identificou maior frequência da AOS em função da idade, observando a ocorrência de IAH $\geq 10 \mathrm{ev} / \mathrm{h}$ associado a sintomas (sonolência diurna, hipertensão ou outra complicação cardiovascular) em $3,3 \%$ na amostra de homens adultos em geral, com prevalência máxima entre indivíduos com 45 a 64 anos de idade $(4,7 \%)$, correspondendo ao aumento de quase quatro vezes em relação à prevalência na faixa etária de 20 a 44 anos de idade $(1,2 \%)$, quando analisada por subgrupos ${ }^{24}$. Em outro estudo, encontrou-se forte associação entre idade e distúrbios respiratórios do sono em ambos os $\operatorname{sexos}^{18}$. Entre homens com sobrepeso, $18,3 \%$, entre 30 a 49 anos de idade, apresenta$\mathrm{ram} \mathrm{IAH} \geq 5 \mathrm{ev} / \mathrm{h}$, enquanto $36,6 \%$, entre 50 a 70 anos, apresentaram esse IAH com uma elevação da frequência em duas vezes. Já entre mulheres, o aumento chegou a aproximadamente cinco vezes nessas faixas etárias: prevalências estimadas em $4,2 \%$ vs. $20,2 \%$, respectivamente ${ }^{18}$.

Um dos estudos analisados ${ }^{12}$ identificou que os trabalhadores diagnosticados com AOS atuavam na empresa há mais tempo, quando comparados aos que não apresentavam AOS. Metzner e Fischer ${ }^{25}$, utilizando o índice de capacidade para o trabalho (ICT) para avaliar a influência do trabalho sobre a saúde de 43 trabalhadores de turno que assumiam escala fixa de 12 horas, observaram que, quanto maior o tempo de exercício na função, menor o ICT. Estes achados são compatíveis com estudos que sugerem que o tempo de atuação em turnos irregulares ou noturnos pode contribuir para a maior frequência de doenças relacionadas ao trabalho ${ }^{1}$. Além da influência da idade sobre a prevalência da AOS nos trabalhadores de turno, os anos acumulados de exposição ao trabalho de turno também podem estar associados à síndrome.

A frequência da AOS de 14,3\% na amostra de enfermeiras do estudo de Geiger-Brown et al..$^{13}$, superior ao estimado para a população feminina em geral, pode ter sido influenciada não apenas pela condição de trabalho de turno, mas também pela faixa etária das enfermeiras participantes, com possível associação com a menopausa. $\mathrm{Na}$ literatura, diversos estudos apontam a influência de alterações hormonais sobre a qualidade do sono ${ }^{26-28}$.

Dancey et al. ${ }^{26}$, comparando mulheres pré e pós-menopausadas, identificaram maior prevalência da AOS em pós-menopausadas, mesmo quando realizada análise por subgrupo de gravidade da doença. Observaram também que o IAH médio das mulheres menopausadas era significativamente maior do que das pré-menopausadas ${ }^{26}$. Young et al. ${ }^{27}$ investigaram distúrbios respiratórios do sono em 589 mulheres que participavam do Wisconsin Sleep Cohort Study, identificando risco significativo associado à transição menopáusica, chegando a 3,5 vezes maior para IAH $\geq 15 \mathrm{ev} / \mathrm{h}$, em mulheres pós-menopausadas, quando comparadas às que não passaram pela menopausa. Bixler et al. ${ }^{28}$ observaram que, enquanto a prevalência da AOS tendia a valores mais baixos em mulheres pré-menopausadas $(0,6 \%)$, a prevalência da doença em pós-menopausadas chegou a 2,7\%, indicando a importância da menopausa enquanto fator de risco para mulheres. Os autores identificaram ainda que, no subgrupo das mulheres pós-menopausadas em tratamento com reposição hormonal, a prevalência da AOS era menor que no grupo daquelas não tratadas $(0,5 \%)$, reforçando a hipótese da influência hormonal no desenvolvimento da doença ${ }^{28}$.

A influência do gênero sobre o processo de saúde e doença também pode impactar na qualidade do sono das mulheres que trabalham de turnos, considerando-se que elas geralmente assumem uma dupla jornada, buscando atender tanto as demandas profissionais, quanto às domésticas ${ }^{1,29}$. Em comparação com os homens, trabalhadoras de turno podem encontrar maiores dificuldades para conciliar trabalho, responsabilidades domésticas e sono, sobretudo no caso daquelas que possuem filhos, como observado por Rotenberg et al. ${ }^{29}$. A dupla ou tripla jornada de trabalho podem estar associadas aos transtornos do sono e perda de capacidade para o traba- 
lho entre as mulheres ${ }^{30}$, envolvendo tanto aspectos biológicos, quanto socioculturais ${ }^{1}$. Estes fatores também podem ter contribuído para maior prevalência da AOS nas enfermeiras estudadas, quando comparada à estimada para as mulheres da população em geral.

Considerando-se os valores de IMC dos trabalhadores de turno, na presente revisão sistemática, identificou-se a presença de sobrepeso e obesidade nas amostras. Quase 70\% dos participantes da pesquisa de Koyama et al. ${ }^{12}$ apresentaram sobrepeso e obesidade, com maior prevalência entre trabalhadores com AOS do que entre os não diagnosticados com a doença, bem como IMC dos trabalhadores de turno com AOS superior em relação ao IMC da totalidade da amostra. Os resultados sugerem que a prevalência da AOS, enquanto condição que guarda estreita relação com obesidade ${ }^{12,18}$, pode também sofrer influência da organização da escala de trabalho.

A prevalência da AOS tem se elevado nas últimas décadas, o que pode estar associado a diversos fatores, sobretudo ao aumento da obesidade na população geral, condição notadamente reconhecida como um dos principais fatores de risco para a doençá ${ }^{18,31}$. O trabalho de turno também tem sido associado ao risco aumentado para sobrepeso e obesidade ${ }^{31,32}$, o que pode estar relacionado às variações no ciclo sono-vigília e jejum-alimentação, com consequente descompasso dos processos circadianos, fisiológicos e metabólicos ${ }^{32}$. Além disso, variáveis como sexo, tempo de exposição, turno de trabalho e fatores psicossociais podem contribuir para o incremento do $\mathrm{IMC}^{33-36}$. Na presente revisão sistemática, observou-se que não só a prevalência da AOS é elevada nos trabalhadores de turno, como também se destaca naqueles que apresentam IMC mais elevado. A interação entre obesidade e trabalho de turno, portanto, pode ter efeitos cumulativos e sobrepostos, aumentando o risco para AOS e potencializando os impactos negativos da doença nessa população.

Nesta revisão sistemática, observou-se a variação das escalas de trabalho ao longo das 24 horas. Estudos apontam para a influência dos horários de trabalho sobre a manifestação da AOS, e o regime que inclui horários noturnos pode associar-se à maior gravidade em pacientes diagnosticados com a doença $a^{37,38}$. Comparando os resultados dos estudos polissonográficos de 31 trabalhadores de turno com AOS realizados durante a noite com aqueles realizados durante $\mathrm{o}$ dia, Paciorek et al..$^{37}$ observaram valores de IAH médio consideravelmente maiores no exame diurno após a noite de trabalho, passando de 31,8 $\pm 21,9$ ev/h na polissonografia noturna para 49,7 $\pm 26,6 \mathrm{ev} / \mathrm{h}$ na polissonografia diurna $(\mathrm{p}<0,05)$.

Resultados semelhantes foram observados em um estudo com oito policiais diagnosticados com AOS, trabalhando em turnos ${ }^{38}$. Apesar de não identificarem impacto relevante do trabalho em horário noturno no IAH de policiais com AOS leve, no subgrupo de trabalhadores com diagnóstico de maior IAH, os autores observaram aumento significativo de eventos obstrutivos por hora de sono, após uma noite de trabalho, sugerindo que, em pacientes com AOS mais grave, a privação aguda de sono pode associar-se à gravidade da doençç ${ }^{38}$.

Trabalhadores com jornadas prolongadas, horários irregulares ou em turnos estão expostos ao risco aumentado de adoecimento e redução da capacidade funcional, sob influência de fatores biológicos e psicossociais ${ }^{1,39}$. Possíveis impactos negativos dessa forma de organização do trabalho podem ser minimizados, a partir de estratégias, como adequação das escalas e tempo de serviço, para conciliar ao máximo as demandas organizacionais e dos trabalhadores ${ }^{1}$. A associação entre trabalho de turno, distúrbios do sono, síndrome metabólica e outras doenças reforça a necessidade do acompanhamento médico desses profissionais ${ }^{1,39}$, incluindo a polissonografia. A prevalência da AOS observada em trabalhadores de turno sinaliza a importância de se considerar o risco para a doença, nessa população, possibilitando seu diagnóstico precoce e intervenção efetiva, com foco na saúde ocupacional.

A heterogeneidade dos estudos, referente aos critérios diagnósticos da AOS, características das amostras e variáveis apresentadas, foi uma limitação da presente revisão sistemática, restringindo a análise comparativa entre os trabalhos. O número reduzido de artigos identificados aponta para a necessidade de pesquisas adicionais sobre a prevalência dessa doença em trabalhadores de turno, com amostras maiores e critérios metodológicos claramente definidos, para determinar com maior segurança a relação entre AOS e fatores relacionados ao trabalho de turno.

Na presente revisão sistemática, identificou-se elevada prevalência da apneia obstrutiva do sono em trabalhadores de turno, comparada à população em geral, sugerindo associação importante entre essa doença e o trabalho de turno, tendo como consequência: maior predisposição para obesidade, hipertensão arterial sistêmica e eventos cardiovasculares. Isto reforça a necessidade de prevenção, identificação e intervenção precoces sobre os potenciais danos à saúde destes trabalhadores. 


\section{Colaboradores}

YS Sakamoto, F Porto-Sousa e C Salles participaram substancialmente da concepção, planejamento, coleta, análise e interpretação dos dados da pesquisa, bem como da elaboração, revisão crítica e aprovação da versão final do manuscrito.

\section{Referências}

1. Moreno RC, Fischer FM, Rotenberg L. A saúde do trabalhador na sociedade 24 horas. São Paulo em Perspect [Internet]. 2003 [cited 2014 Oct 20];17(1):3446. Available from: http://dx.doi.org/10.1590/S010288392003000100005

2. Centers for Disease Control and Prevention (CDC). CDC - Work Schedules: Shift Work and Long Hours NIOSH Workplace Safety and Health Topics [Internet]. 2015 [cited 2015 Aug 19]. Available from: http://www. cdc.gov/niosh/topics/workschedules/

3. Rajaratnam SMW, Howard ME, Grunstein RR. Sleep loss and circadian disruption in shift work: health burden and management. Med J Aust [Internet]. 2013 Oct 21 [cited 2014 Oct 9];199(8):S11-S15. Available from: http://www.ncbi.nlm.nih.gov/pubmed/24138359

4. Pack AI. Advances in sleep-disordered breathing. Am J Respir Crit Care Med [Internet]. 2006 Jan 1 [cited 2015 Sep 23];173(1):7-15. Available from: http://www.ncbi. nlm.nih.gov/pubmed/16284108

5. Wolk R, Somers VK. Sleep and the metabolic syndrome. Exp Physiol [Internet]. 2007 Jan [cited 2014 Oct 9];92(1):67-78. Available from: http://www.ncbi.nlm. nih.gov/pubmed/17085678

6. Floras JS. Sleep apnea and cardiovascular risk. J Cardiol [Internet]. 2014 Jan [cited 2014 Oct 11];63(1):38. Available from: http://www.ncbi.nlm.nih.gov/ pubmed/24084492

7. Müller MR, Guimarães SS. Impacto dos transtornos do sono sobre o funcionamento diário e a qualidade de vida. Estud Psicol [Internet]. 2007 [cited 2014 Oct 18];24(4):519-528. Available from: http://dx.doi. org/10.1590/S0103-166X2007000400011

8. Malta M, Cardoso LO, Bastos FI, Magnanini MMF, Silva CMFP. Iniciativa STROBE : subsídios para a comunicação de estudos observacionais. Rev Saude Publica [Internet]. 2010 [cited 2014 Sep 12];44(3):559-565. Available from: http://dx.doi.org/10.1590/S0034-8910 2010000300021

9. Liberati A, Altman DG, Tetzlaff J, Mulrow C, Gøtzsche PC, Ioannidis JPA, Clarke M, Devereaux PJ, Kleijnen J, Moher D. The PRISMA statement for reporting systematic reviews and meta-analyses of studies that evaluate healthcare interventions: explanation and elaboration. $B M J$ [Internet]. 2009 Jan [cited 2014 Jul 10];339:b2700. Available from: http://www.pubmedcentral.nih. gov/articlerender.fcgi?artid=2714672\&tool=pmcentrez\&rendertype $=$ abstract

10. Santos EHR, Mello MT, Pradella-Hallinan M, Luchesi L, Pires MLN, Tufik S. Sleep and Sleepiness among Brazilian Shift-Working Bus Drivers. Chronobiol Int [Internet]. 2004 [cited 2015 Jan 20];21(6):881-888. Available from: http://www.ncbi.nlm.nih.gov/pubmed $/ 15646235$

11. Klawe JJ, Laudencka A, Mikowiec I, Tafil-Klawe M. Occurence of obstructive sleep apnea in a group of shift worked police officers. J Physiol Pharmacol [Internet]. 2005 [cited 2015 Feb 20];56(Supl. 4):115-117. Available from: http://www.ncbi.nlm.nih.gov/pubmed /16204784 
12. Koyama RG, Esteves AM, Oliveira e Silva L, Lira FS, Bittencourt LRA, Tufik S, Mello MT. Prevalence of and risk factors for obstructive sleep apnea syndrome in Brazilian railroad workers. Sleep Med [Internet]. 2012 Sep [cited 2015 Feb 20];13(8):1028-1032. Available from: http://www.ncbi.nlm.nih.gov/pubmed/22841037

13. Geiger-Brown J, Rogers VE, Han K, Trinkoff A, Bausell $\mathrm{RB}$, Scharf SM. Occupational screening for sleep disorders in 12-h shift nurses using the Berlin Questionnaire. Sleep Breath [Internet]. $2013 \mathrm{Mar}$ [cited $2015 \mathrm{Feb}$ 23];17(1):381-388. Available from: http://www.ncbi. nlm.nih.gov/pubmed/22535196

14. Geiger-Brown J, Rogers VE, Trinkoff AM, Kane RL, Bausell RB, Scharf SM. Sleep, sleepiness, fatigue, and performance of 12-hour-shift nurses. Chronobiol Int [Internet]. 2012 Mar [cited 2015 Jun 25];29(2):211219. Available from: http://www.ncbi.nlm.nih.gov/ pubmed/22324559

15. Ma J, Zhang C, Zhang J, Hu J, Fang J, Zhang J, Jia P, Wang G. Prospective study of first night effect on 2-night polysomnographic parameters in adult Chinese snorers with suspected obstructive sleep apnea hypopnea syndrome. Chin Med J [Internet]. 2011 [cited 2016 Jul 20];124(24):4127-4131. Available from: http://124.205.33.103:81/ch/reader/view_abstract.aspx?file_no $=2011121938354630 \& f l a g=1$

16. Young T, Palta M, Dempsey J, Skatrud J, Weber S, Badr $\mathrm{S}$. The occurrence of sleep-disordered breathing among middle-aged adults. N Engl J Med [Internet]. $1993 \mathrm{Apr}$ 29 [cited 2015 Aug 20];328(17):1230-1235. Available from: http://www.ncbi.nlm.nih.gov/pubmed/8464434

17. Kim J, In K, Kim J, You S, Kang K, Shim J, Lee S, Lee J, Lee S, Park C, Shin C. Prevalence of sleep-disordered breathing in middle-aged Korean men and women. Am J Respir Crit Care Med [Internet]. 2004 Nov 15 [cited 2015 Aug 23];170(10):1108-1113. Available from: http://www.ncbi.nlm.nih.gov/pubmed/15347562

18. Peppard PE, Young T, Barnet JH, Palta M, Hagen EW, Hla KM. Increased prevalence of sleep-disordered breathing in adults. Am J Epidemiol [Internet]. 2013 May 1 [cited 2015 Feb 12];177(9):1006-1014. Available from: http://www.pubmedcentral.nih.gov/articlerender.fcgi?artid=3639722\&tool=pmcentrez\&rendertype $=$ abstract

19. Epstein LJ, Kristo D, Strollo PJ, Friedman N, Malhotra A, Patil SP, Ramar K, Rogers R, Schwab RJ, Weaver EM, Weinstein MD. Clinical Guideline for the Evaluation, Management and Long-term Care of Obstructive Sleep Apnea in Adults. J Clin Sleep Med [Internet]. 2009 [cited 2015 Aug 20];5(3):263-276. Available from: http://www.aasmnet.org/Resources/clinicalguidelines/ OSA_Adults.pdf

20. Berry RB, Budhiraja R, Gottlieb DJ, Gozal D, Iber C Kapur VK, Marcus CL, Mehra R, Parthasarathy S, Quan SF, Redline S, Strohl KP, Ward SLD, Tangredi MM. Rules for scoring respiratory events in sleep: update of the 2007 AASM Manual for the Scoring of Sleep and Associated Events. Deliberations of the Sleep Apnea Definitions Task Force of the American Academy of Sleep Medicine. J Clin Sleep Med [Internet]. 2012 Oct 15 [cited 2015 Jun 26];8(5):597-619. Available from: http://www.pubmedcentral.nih.gov/articlerender.fcgi?artid $=3459210 \&$ tool $=$ pmcentrez\&rendertype $=a b-$ stract
21. Tufik S, Santos-Silva R, Taddei JA, Bittencourt LRA Obstructive sleep apnea syndrome in the Sao Paulo Epidemiologic Sleep Study. [Internet]. Sleep medicine. 2010 [cited 2015 Aug 20];11(5):441-446. Available from: http://dx.doi.org/10.1016/j.sleep.2009.10.005

22. Bruschini MCA. Trabalho e gênero no Brasil nos últimos dez anos. Cad Pesqui [Internet]. 2007 [cited 2015 Feb 20];37(132):537-572. Available from: http://dx.doi. org/10.1590/S0100-15742007000300003

23. Punjabi NM. The epidemiology of adult obstructive sleep apnea. Proc Am Thorac Soc [Internet]. $2008 \mathrm{Feb}$ 15 [cited 2015 Feb 19];5(2):136-143. Available from: http://www.pubmedcentral.nih.gov/articlerender.fcgi?artid $=2645248 \&$ tool $=$ pmcentrez\&rendertype $=a b-$ stract

24. Bixler EO, Vgontzas AN, Have TT, Tyson K, Kales A. Effects of age on sleep apnea in men: I. Prevalence and severity. Am J Respir Crit Care Med [Internet]. 1998 Jan [cited 2015 Aug 20];157(1):144-148. Available from: http://www.ncbi.nlm.nih.gov/pubmed/9445292

25. Metzner RJ, Fischer FM. Fadiga e capacidade para o trabalho em turnos fixos de doze horas. Rev Saude Publica [Internet]. 2001 Dec [cited 2015 Oct 31];35(6):548-553. Available from: http:// www. scielo.br/scielo.php?script $=$ sci_arttext\&pid $=$ S0034 $-89102001000600008 \& \operatorname{lng}=\mathrm{pt} \& \mathrm{nrm}=\mathrm{iso} \& \operatorname{lng}=\mathrm{pt}$

26. Dancey D, Hanly P, Soong C, Lee B, Hoffstein V. Impact of menopause on the prevalence and severity of sleep apnea. Chest [Internet]. 2001 Jul 1 [cited 2015 Oct 31];120(1):151-155. Available from: http://journal. publications.chestnet.org/article.aspx?doi $=10.1378 /$ chest.120.1.151

27. Young T, Finn L, Austin D, Peterson A. Menopausal status and sleep-disordered breathing in the Wisconsin Sleep Cohort Study. Am J Respir Crit Care Med [Internet]. 2003 May 1 [cited 2015 Oct 31];167(9):11811185. Available from: http://www.ncbi.nlm.nih.gov/ pubmed/12615621

28. Bixler EO, Vgontzas AN, Lin H, Have TT, Rein J, Vela-Bueno A, Kales A. Prevalence of sleep-disordered breathing in women: effects of gender. Am J Respir Crit Care Med [Internet]. 2001 Mar [cited 2015 Oct 31];163(3 Pt 1):608-613. Available from: http://www. ncbi.nlm.nih.gov/pubmed/11254512

29. Rotenberg L, Portela LF, Marcondes WB, Moreno C, Nascimento CP. Gênero e trabalho noturno: sono, cotidiano e vivências de quem troca a noite pelo dia. Cad Saude Publica [Internet]. 2001 Jun [cited 2015 Nov 01];17(3):639-649. Available from: http://www.scielo.br/scielo.php?script=sci_arttext\&pid=S0102-311X2001000300018\&lng=pt\&n$\mathrm{rm}=\mathrm{iso} \& \mathrm{t} \operatorname{lng}=\mathrm{pt}$

30. Fischer FM, Borges NS, Rotenberg L, Latorre MRDO, Soares NS, Rosa PLFS, Nagai R, Landsbergis P. A (in) capacidade para o trabalho em trabalhadores de enfermagem. Rev Bras Med Trab [Internet]. 2005 [cited 2015 Oct 31]; 3(2):97-103. Available from: http:// www.anamt.org.br/site/upload_arquivos/revista brasileira_de_medicina_do_trabalho_-_volume_3 20122013151036533424.pdf 
31. Garvey JF, Pengo MF, Drakatos P, Kent BD. Epidemiological aspects of obstructive sleep apnea. J Thorac Dis [Internet]. 2015 May [cited 2015 Jun 25];7(5):920929. Available from: http://www.ncbi.nlm.nih.gov/ pubmed/26101650

32. Zimberg IZ, Fernandes Júnior SA, Crispim CA, Tufik S, Mello MT. Metabolic impact of shift work. Work [Internet]. 2012 Jan [cited 2014 Oct 11];41 Suppl 1:43764383. Available from: http://www.ncbi.nlm.nih.gov/ pubmed/22317392

33. Antunes LC, Levandovski R, Dantas G, Caumo W, Hidalgo MP. Obesity and shift work: chronobiological aspects. Nutr Res Rev [Internet]. 2010 Jun [cited 2014 Oct 6];23(1):155-168. Available from: http://www.ncbi. nlm.nih.gov/pubmed/20122305

34. Griep RH, Bastos LS, Fonseca MJM, Silva-Costa A, Portela LF, Toivanen S, Rotenberg L. Years worked at night and body mass index among registered nurses from eighteen public hospitals in Rio de Janeiro, Brazil. BMC Health Serv Res [Internet]. 2014 Jan [cited 2015 Aug 6];14:603. Available from: http://www.pubmedcentral. nih.gov/articlerender.fcgi?artid $=4264337 \&$ tool $=$ pmcentrez\&rendertype $=$ abstract

35. Ishizaki M, Morikawa Y, Nakagawa H, Honda R, Kawakami N, Haratani T, Kobayashi F, Araki S, Yamada Y. The Influence of Work Characteristics on Body Mass Index and Waist to Hip Ratio in Japanese Employees. Ind Health [Internet]. 2004 [cited 2015 Aug 20];42(1):41-49. Available from: http://joi.jlc.jst.go.jp/ JST.Journalarchive/indhealth1963/42.41?from=CrossRef
36. Peplonska B, Bukowska A, Sobala W. Association of Rotating Night Shift Work with BMI and Abdominal Obesity among Nurses and Midwives. PLoS One [Internet]. 2015 Jan [cited 2015 Aug 6];10(7):e0133761. Available from: http://www.pubmedcentral.nih.gov/ articlerender.fcgi artid $=4511417 \&$ tool $=$ pmcentrez\& rendertype $=$ abstract

37. Paciorek M, Korczyński P, Bielicki P, Byśkiniewicz K, Zieliński J, Chazan R. Obstructive sleep apnea in shift workers. Sleep Med [Internet]. 2011 Mar [cited 2014 Oct 9];12(3):274-277. Available from: http://www.ncbi. nlm.nih.gov/pubmed/21316298

38. Laudencka A, Klawe JJ, Tafil-Klawe M, ZEomañczuk P. Does night-shift work induce apnea events in obstructive sleep apnea patients? J Physiol Pharmacol [Internet]. 2007 [cited 2015 Jun 20];58(1):345347. Available from: http://www.ncbi.nlm.nih.gov/ pubmed/18204146

39. Costa G. Shift work and occupational medicine: an overview. Occup Med [Internet]. 2003 Mar 1 [cited 2015 Sep 24];53(2):83-88. Available from: http://occmed.oupjournals.org/cgi/doi/10.1093/occmed/kqg045

Artigo apresentado em 17/03/2016

Aprovado em 21/10/2016

Versão final apresentada em 23/10/2016 